\title{
Palliative Electrochemotherapy Treatment of Cutaneous Metastases in a Patient with Advanced Thyroid Papillary Carcinoma
}

\author{
Daniela Cavaco $^{\mathrm{a}}$ Sara Carvalhal ${ }^{\mathrm{b}}$ Valeriano Leite ${ }^{\mathrm{a}, \mathrm{c}}$

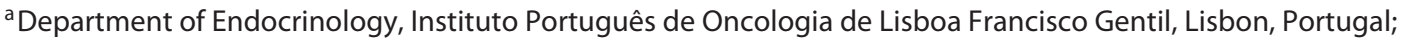 \\ ${ }^{b}$ Department of General Surgery, Instituto Português de Oncologia de Lisboa Francisco Gentil, Lisbon, Portugal; \\ 'NOVA Medical School/Faculdade de Ciências Médicas, Universidade Nova de Lisboa, Lisbon, Portugal
}

\section{Established Facts}

- Electrochemotherapy (ECT) is a minimally invasive oncologic treatment of tumors located in the skin and subcutaneous tissue and is highly effective especially to relieve pain, stop bleeding, and improve the quality of life.

- Metastatic papillary thyroid carcinoma (PTC) with cutaneous metastases may cause pain, ulceration, and bleeding.

\section{Novel Insights}

- ECT seems to be a safe and effective technique and may be an option for local palliative treatment in cutaneous metastases of PTC with improvement of local symptoms.

\section{Keywords}

Advanced thyroid cancer - Electrochemotherapy ·

Cutaneous metastases $\cdot$ Case report

\section{Abstract}

Advanced papillary thyroid carcinoma (PTC) with cutaneous metastases may cause pain, ulceration, and bleeding. Electrochemotherapy (ECT) is a minimally invasive treatment of tumors located in the skin and subcutaneous tissue. The electric pulses potentiate the toxicity of cytostatic agents en- tering the tumor cell. It is highly effective especially to relieve pain and improve the quality of life. The adverse events are local and transient. A case of progressive metastatic PTC who developed bleeding cutaneous metastases treated with ECT is described.

(C) 2020 European Thyroid Association Published by S. Karger AG, Basel

This case report was presented as an oral presentation at the "71st Annual Meeting of the Portuguese Society of Endocrinology, Diabetes and Metabolism," Coimbra, Portugal, January 23-27, 2020.
Daniela Cavaco

Department of Endocrinology Instituto Português de Oncologia de Lisboa Francisco Gentil, Rua Prof. Lima Basto PT-1099-023 Lisbon (Portugal)

daniela.rcavaco@gmail.com 


\section{Introduction}

The vast majority of thyroid cancers of follicular origin have a very favorable outcome, but $5-10 \%$ of the cases will develop metastatic disease [1]. The most common sites of metastatic differentiated thyroid cancer are the neck lymph nodes, lungs, bones, and less frequently the brain [2]. The skin is an uncommon metastatic site of thyroid cancer but, when it occurs, the scalp is the most frequent region involved $[2,3]$. Cutaneous metastases may cause pain, ulceration, and bleeding as well as anxiety due to the awareness of tumor growth [4]. Electrochemotherapy (ECT) has been developed over the past decade as a minimally invasive oncologic treatment. The electric pulses used by ECT promote a transient increase in the permeability of cell membranes, which potentiates toxicity of a cytostatic agent entering the tumor cell $[5,6]$. It is effective, especially in the palliative treatment of cancers located in the skin and subcutaneous tissue, and is usually applied in the palliative settings for the treatment of patients with unresectable tumors to relieve pain and improve the quality of life $[7,8]$. ECT is an effective treatment for both primary tumors and their metastases, regardless of the histological type and previous treatments. It has also shown its effectiveness in the case of treating deeply located tumors [9]. ECT is not recommended in patients with cardiac pacemakers and patients on anticoagulant therapy for safety reasons [9]. A case of progressive metastatic papillary thyroid carcinoma (PTC) who developed bleeding cutaneous metastases treated with ECT is described.

\section{Case Presentation}

A 85-year-old male with a history of coronary disease, chronic renal failure stage 4 , renal lithiasis complicated with hydronephrosis, prostate carcinoma with biochemical evidence of disease, and bilateral hip arthroplasty, reported the appearance, 18 months before, of a neck nodule with indolent growth and multiple cutaneous lesions in the scalp which had previously been submitted to surgical excision in another institution. Histology of the skin lesions was not available.

The patient was referred to our center to investigate the cervical nodule, which was a hard, but not adherent, supraclavicular node $5 \mathrm{~cm}$ in diameter. There was no evidence of compressive signs such as dysphagia or dyspnea. Thyroid gland palpation revealed a soft nodule of $1.5 \mathrm{~cm}$ in the left lobe. The patient also complained of lumbar pain with radiation to the lower limbs with decreased muscle strength bilaterally. There were no signs or symptoms of thyroid dysfunction.

Neck ultrasound revealed multiple hypoechogenic and solid nodules with macrocalcifications, the largest with $13 \mathrm{~mm}$, in the thyroid gland and a heterogeneous and calcified nodule of $52 \times 44$ $\times 21 \mathrm{~mm}$ located in the right supraclavicular region. Fine-needle aspiration of a thyroid nodule and the supraclavicular nodule was suggestive of PTC. Thyroid function was normal, thyroperoxidase antibodies were in the normal range, and serum thyroglobulin was $971 \mathrm{ng} / \mathrm{mL}$ (normal range 0.2-55). A computed tomography (CT) revealed an adenopathic conglomerate of $51 \times 39 \mathrm{~mm}$ located in the right supraclavicular region with extension to the superior mediastinum and an extensive lytic lesion involving the vertebral bodies of L3 and L4 with dural extrinsic molding and foraminal stenosis. The fluorodeoxyglucose positron emission tomography revealed an extensive metastization into cervical lymph nodes, bone, lung, and liver. Due to the advanced age of the patient, extensive metastatic disease of thyroid cancer and multiple comorbidities, a multidisciplinary team decided for palliative treatment, and symptomatic spinal bone metastases were treated with palliative radiothera-
Fig. 1. The scalp metastasis on a CT scan (a) and the correspondent fluorodeoxyglucose positron emission tomography image (b).
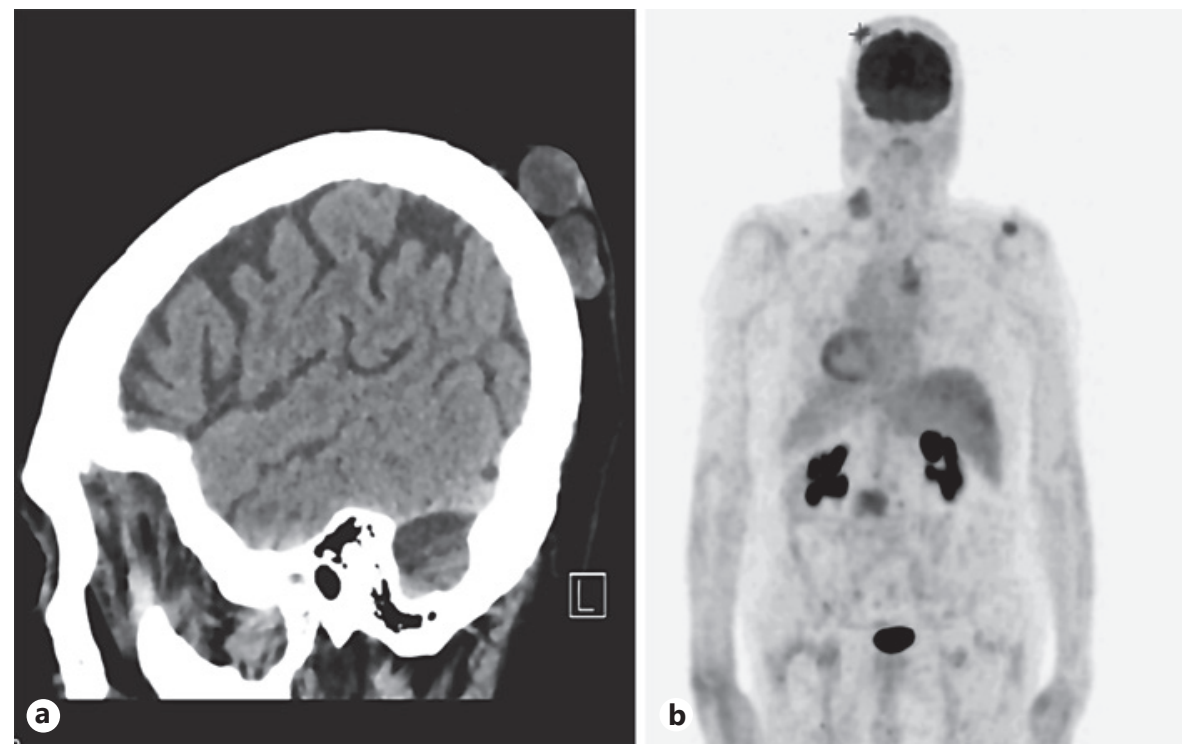
py with $20 \mathrm{~Gy}$ in 5 fractions. In the follow-up, the patient developed new and multiple, firm, non-painful scalp nodules, the largest being $20 \mathrm{~mm}$. A CT scan excluded bone invasion of the skull (Fig. 1a, b). Fine-needle aspiration of the scalp lesions revealed PTC metastases, and thyroglobulin was $>30.000 \mathrm{ng} / \mathrm{mL}$ in needle washout fluid. Later on, the lesions developed active bleeding and did not respond to local treatment with silver nitrate (Fig. 2a).

The patient was submitted to an ECT session with intravenous bleomycin $\left(15,000 \mathrm{UI} / \mathrm{m}^{2}\right)$ administered in a bolus prior to the administration of electroporation pulses targeting the described lesion. The electroporation device was the Cliniporator ${ }^{\circledR}$ (IGEA), which allows the application of electric pulses via sets of needle electrodes with computed monitoring to prevent excess current for patient safety. The procedure took place in the ambulatory surgery unit under general anesthesia without complications. The bleeding stopped in the following days (Fig. 2b). The procedure was well tolerated except for mild local pruritus, and the patient did not develop other complications. A clinical response was observed, without new episodes of active bleeding in the scalp during a follow-up of 10 months (Fig. 2c).

\section{Discussion and Conclusion}

ECT was successfully used to treat bleeding scalp metastases in this patient who had advanced thyroid cancer and serious comorbidities and was not a candidate for aggressive surgical or medical (i.e., kinase inhibitors) treatments. Conventional radiotherapy could have been considered because of its local hemostatic effect; however, this form of therapy was not readily available at our insti- tution and metastases were actively bleeding, imposing urgent care. ECT has the advantage of being performed in an ambulatory surgical unit with only half-day to 1 day of hospital stay and can be repeated several times. Since the most common ECT adverse events are local (moderate pain, erythema, swelling, and muscle contractions) and transient, this technique seems to be a safe and effective option for local palliative treatment of cutaneous metastases of thyroid cancer with improvement of local symptoms.

\section{Statement of Ethics}

The authors declare that the procedures were followed according to the regulations established by the Clinical Research and Ethics Committee and to the Helsinki Declaration of the World Medical Association. The authors declare that the manuscript, complete or in parts, does not infringe any copyright and does not violate any privacy rights. The authors declare that no experiments were performed on humans or animals for this investigation. The authors state that the subject has given his written informed consent to publish his case, including publication of images.

\section{Disclosure Statement}

The authors have no conflicts of interests to declare.
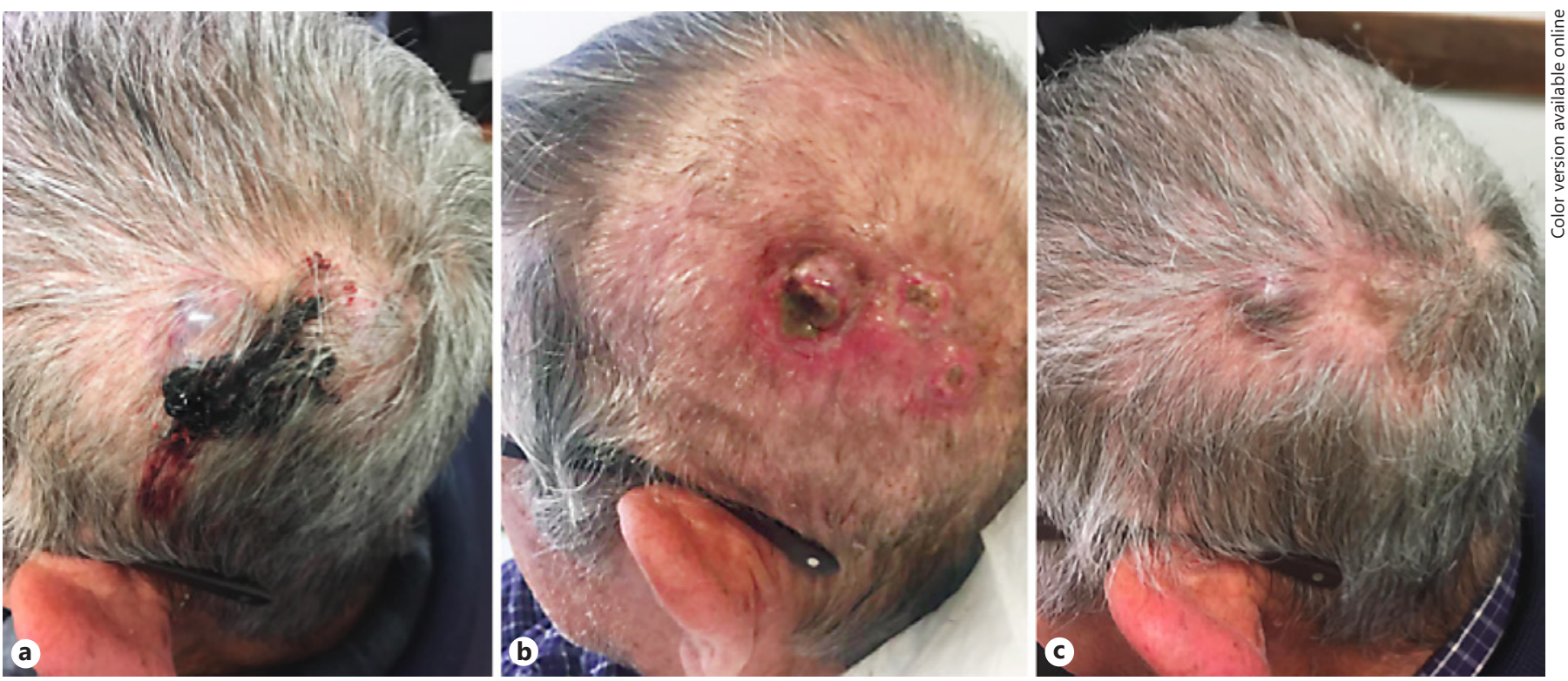

Fig. 2. Evolution of the skin metastasis before (a) and 1 week (b) and 3 months (c) after ECT.

Palliative Electrochemotherapy Treatment in Advanced Thyroid Cancer
Eur Thyroid J 2020;9:221-224

DOI: $10.1159 / 000507888$ 


\section{Funding Sources}

The authors received no financial support for the research, authorship, and/or publication of this article.

\section{Author Contributions}

All authors made substantial contributions to this study, and all authors approved the final version of the manuscript. The authors declare that the manuscript is not under review of any other journal, and that it has not been published completely or partially in any other journal.

\section{References}

1 De Virgilio A, Ralli M, Longo L, Mancini P, Attanasio G, Atturo F, et al. Electrochemotherapy in head and neck cancer: A review of an emerging cancer treatment [Review]. Oncol Lett. 2018 Sep;16(3):3415-23.

2 Grau JJ, Caballero M, Langdon C, BernalSprekelsen M, Blanch JL. Electrochemotherapy as palliative treatment in patients with thyroid papillary carcinoma. Braz J Otorhinolaryngol. 2016 May-Jun;82(3):285-8.

3 Lenzi R, Muscatello L, Saibene AM, Felisati G, Pipolo C. The controversial role of electrochemotherapy in head and neck cancer: a systematic review of the literature. Eur Arch Otorhinolaryngol. 2017 Jun;274(6):2389-94.
4 Farina E, Monari F, Tallini G, Repaci A, Mazzarotto R, Giunchi F, et al. Unusual Thyroid Carcinoma Metastases: a Case Series and Literature Review. Endocr Pathol. 2016 Mar; 27(1):55-64.

5 Morley J, Grocott P, Purssell E, Murrells T. Electrochemotherapy for the palliative management of cutaneous metastases: A systematic review and meta-analysis. Eur J Surg Oncol. 2019 Dec;45(12):2257-67.

6 Probst U, Fuhrmann I, Beyer L, Wiggermann P. Electrochemotherapy as a New Modality in Interventional Oncology: A Review. Technol Cancer Res Treat. 2018 Jan; 17: 1533033818785329.
7 Tumino D, Frasca F, Newbold K. Updates on the Management of Advanced, Metastatic, and Radioiodine Refractory Differentiated Thyroid Cancer. Front Endocrinol (Lausanne). 2017 Nov;8:312.

8 Aghasi MR, Valizadeh N, Soltani S. A 64 yearold female with scalp metastasis of papillary thyroid cancer. Indian J Endocrinol Metab. 2011 Jul;15(6 Suppl 2):S136-7.

9 Esmaeili N, Friebe M. Electrochemotherapy: A Review of Current Status, Alternative IGP Approaches, and Future Perspectives. J Healthc Eng. 2019 Jan;2019:2784516. 